\title{
A leitura em crianças prematuras com perturbações neuromotoras
}

\author{
Reading in preterm children with neuro-motor disorders
}

\author{
Iolanda Campos-Gil*, Maria Vânia Nunes** \\ *Psicóloga no Centro de Reabilitação de Paralisia Cerebral Calouste Gulbenkian e Doutoranda em Ciências da Cognição \\ e Linguagem na Universidade Católica Portuguesa, ${ }^{* *}$ Docente na Universidade Católica Portuguesa
}

\begin{abstract}
Resumo
Este trabalho visa avaliar em simultâneo os perfis do processamento visual e fonológico na leitura, em crianças nascidas prematuras com perturbação neuromotora ligeira e moderada. Para avaliação do processamento visual na leitura registou-se o seguimento ocular (eye-tracking), e para avaliação do processamento fonológico considerou-se a análise da voz gravada no teste ALEPE. Constituíram-se 3 grupos de crianças em idade escolar, um de prematuros e outro de termo com perturbação neuromotoras, e um terceiro grupo de crianças tipicamente desenvolvidas. Foram analisados os perfis e encontradas características de leitura específicas revelando-se o grupo de crianças nascidas prematuras de maior fragilidade.
\end{abstract}

Palavras chave: leitura, processamento visual e fonológico, pré-termo, idade escolar.

\section{Introdução}

Os avanços científicos das últimas décadas nos cuidados neonatais sobretudo com a introdução de metodologias de regulação neurocomportamental e hemodinâmica do recém-nascido pré-termo proporcionaram uma sobrevivência cada vez maior desta população. Segundo dados do Instituto Nacional de Estatística em Portugal os prematuros representam $7,8 \%$ dos nados vivos. A paralisia cerebral $\mathrm{e}$ as perturbações do neurodesenvolvimento são as sequelas mais temidas nesta população, estando a prevalência da paralisia cerebral estável à custa dos nascimentos prétermo (Himmelmann, Hagberg \& Uvebrant, 2010; Platt et al, 2007). Esta etiologia representa $45 \%$ dos casos registados aos 5 anos com paralisia cerebral em Portugal. O risco dos nascidos pré-termo virem a desenvolver paralisia cerebral aumenta proporcionalmente relativamente ao grau de prematuridade, quer em peso ao nascer quer em semanas de gestação (Programa de Vigilância da Paralisia Cerebral aos 5 anos de idade - PVNPC). Atualmente o diagnóstico de paralisia cerebral reportase às alterações/perturbações/lesões neurológicas do movimento e da postura comprometendo predominantemente o domínio motor (Bax et al, 2005), mas podendo englobar outros défices em coorbilidade como a cognição (Kerr-Wilson et al 2012), nomeadamente aspetos visuopercetivos, da comunicação e linguagem e consequentemente perturbações da aprendizagem na idade escolar (Anderson et al 2003; Bhutta, Cleves, Casey, Cradock \& Anand 2002; Guarini et al 2010; Litt, Taylor,Klein \& Hack 2005; Kellr-Margulis, Dempsey \& Llorens 2011; Olivieri et al 2012) que podem prevalecer até mais tarde na adolescência e idade adulta (Saigal \& Doyle 2008). A perturbação neuromotora ligeira e moderada, correspondendo aos níveis I, II e III do sistema de classificação da função motora global (Palisano et al 1997; Rosenbaum, Palisano, Bartlett, Galuppi \& Russell 2008) atinge em Portugal 52\% das crianças com paralisia cerebral, segundo o PVNPC aos 5 anos; o nível cognitivo com QI superior a 70 atinge $41 \%$ neste estudo em Portugal. As alterações neuroftalmológicas e visuopercetivas nos prematuros quer a nível cortical, área visual da leitura de palavras, quer a nível da substância branca, têm sido reportadas como um importante fator a influenciar as aprendizagens escolares (Bassi et al, 2008; Butcher et al, 2012; Fazzi et al, 2012; Dehaene et al, 2010; Dehaene \& Cohen, 2011; Narberhaus et al, 2009; O'Reilly et al 2010; Ortibus, De Cock \& Lagae, 2011; Ricci et al, 2010;). Recentemente com o desenvolvimento de tecnologia de ressonância magnética e tractografia são identificadas com melhor precisão alterações estruturais cerebrais nas crianças nascidas pré-termo, com redução do volume global cortical e leucomalácia periventricular a nível subcortical, alterações na espessura do corpo caloso e cápsula interna associados às perturbações do neurodesenvolvimento (Andrews et al, 2009; Anjari et al, 2007; de Bruïne, 2010; Sripada et al, 2015; Volpe, 2009).

A aprendizagem da leitura é uma das primeiras aquisições na idade escolar e por isso constitui um forte indicador das dificuldades cognitivas nesta idade. A leitura de palavras é um processo complexo que para além de implicar o processamento fonológico a nível da linguagem (Downie, Jakobson, Frisk, \& Ushycky , 2002) envolve o processamento cognitivo visuopercetivo e visuomotor (Blythe, Liversedge, Joseph, White \& Rayner, 2009; Dambacher, Slattery, Yang, Kliegl \& Rayner 2013; Nazir \& Huckauf, 2008). A investigação para avaliar a atividade da leitura tem recorrido à tecnologia eye-tracking, sendo esta técnica 
uma forte aliada dos investigadores sobretudo na visualização do padrão de processamento visual ao nível dos aspetos visuo-motores (Hand, O’Donnell \& Sereno, 2012; Rayner, 2009; Rayner, Slattery, Drieghe \& Liversedge, 2011). No entanto nas crianças nem sempre é fácil utilizar esta técnica devido às implicações e exigências decorrentes do desenho experimental em estudos de leitura assim como na calibração e controle postural necessários durante os procedimentos de avaliação. A calibração destes sistemas assim como a montagem do desenho de avaliação constitui a maior dificuldade deste tipo de investigação necessitando experiência do investigador na avaliação desta população. A atenção visual, a velocidade de processamento, o controlo visuo-motor assim como a perceção visuo-espacial são funções que podem ser avaliadas com recurso ao eye-traking e a análise do material pode fornecer pistas para elaborar estratégias e padrões de exploração visual e fonológica na leitura. $\mathrm{O}$ padrão dos movimentos oculares na leitura varia consoante a idade e experiência do leitor e envolve por um lado movimentos de seguimento visual, também chamados sacádicos, percurso num sentido geralmente esquerda-direita, com duração de 20 a 50ms, fixações, que são as pausas de tempo variável e traduzem o tempo necessário para a extração a informação e ainda regressões que são o retorno do movimento a pontos anteriores e podem traduzir a necessidade de reler o material anterior de forma clarificarem a sua compreensão (Ellis, 1995). É durante a fixação ocular que é extraída a informação sobre o que lemos, durando cerca de 200 a 250ms (Spinelli \& Ferrand, 2005). A utilização desta ferramenta digital aliada à avaliação da leitura constitui um forte contributo para a compreensão dos processos de leitura sobretudo em grupos com dificuldades de realização e maior fragilidade neuromotora.

O objetivo deste estudo é fazer uma análise do perfil do processamento visual e fonológico na atividade de leitura numa amostra de crianças nascidas pré-termo e compará-lo com um grupo com idênticas perturbações neuromotoras mas nascido de termo e com um grupo de controlo com crianças tipicamente desenvolvidas.

\section{Metodologia}

\section{Participantes}

O estudo decorreu entre setembro de 2014 e junho de 2015. Na primeira fase recorreu-se à seleção de uma amostra de conveniência recolhida de uma coorte de 156 crianças com idades entre os 7 e os 10 atendidos no Centro de Paralisia Cerebral Calouste Gulbenkian em Lisboa de onde foram retirados dois grupos um (G1) com idade de gestação $<37$ semanas constituído o grupo de crianças nascidas pré-termo $\mathrm{N}=25$ e outro (G2) de crianças nascidas de termo $>=37$ semanas $\mathrm{N}=$ 25 ambos com níveis cognitivos com QI>70 e níveis de perturbação neuromotora ligeira a moderada segundo o Sistema de Classificação da Função Motora (SCFM) e a frequentar o ensino regular $\left(1^{\circ}\right.$ ciclo) com apoios ao abrigo da legislação em vigor em Portugal DL 3/2008 para crianças com necessidades educativas especiais
(NEE). Foi ainda constituído um outro grupo de crianças tipicamente desenvolvidas nascidas de termo sem patologias cognitivas ou neuromotoras e sem NEE a frequentar a escola regular $\mathrm{N}=32$. Os três grupos foram constituídos homogéneos em relação ao sexo, idade frequência escolar e escolaridade materna. Os três grupos são previamente identificados com as provas visuo-percetivas da WISC III - índice percetivo (Wechsler, 2003) e Figura Complexa de Rey (Rey, 2002) e a nível do Desenvolvimento da linguagem com a Grelha de observação da linguagem-Gol-E (Kay \& Santos, 2014) e Wisc III - índice compreensão verbal. $\mathrm{Na}$ última fase no final do ano letivo, foram excluídos 4 elementos do G1 e 5 do G2 por limitações inerentes ao nível de leitura apresentado (sub-silábica) e 4 elementos do G3 faltaram à avaliação ficando assim constituídos os grupos: $\mathrm{G} 1 \mathrm{~N}=21 ; \mathrm{G} 2 \mathrm{~N}=20 ; \mathrm{G} 3 \mathrm{~N}=28$.

Seguem-se as tabelas 1, 2, 3 e 4 com a análise descritiva que caracterizam os dois grupos com perturbação neuromotora ligeira a moderada que frequentam a escola regular embora com necessidades educativas especiais e apoios semanais por técnicos especializados ao abrigo do Decreto Lei 3/2008 da lei portuguesa que regula a inclusão e os apoios em classes regulares.

O G1, de crianças nascidas pré-termo, apresenta ao nascer menor peso em relação ao G2 nascidos de termo, e uma imaturidade com alterações biológicas relacionadas com a prematuridade, conforme tabelas 2,3 e 4 .

Tabela 1

Caraterização dos grupos 1 e 2 quanto ao peso e semanas de gestação

\begin{tabular}{|c|c|c|c|c|c|c|}
\hline & & $\mathrm{N}$ & Min. & Max. & Média & $\begin{array}{l}\text { Desvio } \\
\text { padrão }\end{array}$ \\
\hline \multirow[b]{2}{*}{ G1 } & Peso (g) & 21 & 600 & 2725 & 1453.33 & 704.387 \\
\hline & $\begin{array}{l}\text { Semanas } \\
\text { Gestação }\end{array}$ & 21 & 24 & 36 & 29.71 & 4.113 \\
\hline \multirow[b]{2}{*}{ G2 } & Peso (g) & 20 & 2610 & 4155 & 3144,45 & 387.835 \\
\hline & $\begin{array}{l}\text { Semanas } \\
\text { Gestação }\end{array}$ & 20 & 38 & 41 & 38.95 & 0.887 \\
\hline
\end{tabular}

Tabela 2

Caraterização dos Grupos 1 e 2 em relação ao diagnóstico em frequências e percentagens

\begin{tabular}{lrrrr}
\hline \multirow{2}{*}{ Diagnóstico } & \multicolumn{2}{c}{ G1 } & \multicolumn{2}{c}{ G2 } \\
\cline { 2 - 5 } & $\begin{array}{c}\text { Freq } \\
(\mathrm{n})\end{array}$ & $\begin{array}{c}\text { Perc } \\
(\%)\end{array}$ & $\begin{array}{r}\text { Freq } \\
(\mathrm{n})\end{array}$ & \multicolumn{1}{c}{$\begin{array}{c}\text { Perc } \\
(\%)\end{array}$} \\
\hline ADPM & 7 & 33.3 & 13 & 65.0 \\
ADPM + Atraso Linguagem & 3 & 14.3 & 0 & 0.0 \\
PC Ataxia & 1 & 4.8 & 1 & 5.0 \\
PC Espástica Bilateral & 8 & 38.1 & 1 & 5.0 \\
PC Espástica Unilateral & 2 & 9.5 & 4 & 20.0 \\
PC Atetose & 0 & 0.0 & 1 & 5.0 \\
Total & 21 & 100.0 & 20 & 100.0 \\
\hline
\end{tabular}

ADPM=Atraso do Desenvolvimento Psicomotor;

$\mathrm{PC}=$ paralisia cerebral 
Tabela 3

Alterações/lesões cerebrais reportadas em ressonância magnética no G1 e G2 em frequências e percentagens

\begin{tabular}{|c|c|c|c|c|}
\hline \multirow{2}{*}{$\begin{array}{c}\text { RM } \\
\text { Ressonância magnética }\end{array}$} & \multicolumn{2}{|c|}{ G1 } & \multicolumn{2}{|c|}{ G2 } \\
\hline & $\begin{array}{l}\text { Freq } \\
(\mathrm{n})\end{array}$ & $\begin{array}{l}\text { Perc } \\
(\%)\end{array}$ & $\begin{array}{l}\text { Freq } \\
(\mathrm{n})\end{array}$ & $\begin{array}{l}\text { Perc. } \\
(\%)\end{array}$ \\
\hline Lesões Cerebelo & 1 & 4.8 & 1 & 5.0 \\
\hline LPV & 10 & 47.6 & 0 & 0.0 \\
\hline LPV+ Lesões Corticais & 1 & $4, .8$ & 3 & 15.0 \\
\hline Malformações & 2 & 9.5 & 1 & 5.0 \\
\hline LPV + Lesões Talâmicas & 0 & 0 & 2 & 10.0 \\
\hline LPV+ Corpo Caloso & 0 & 0 & 1 & 5.0 \\
\hline Sem Alterações RM & 0 & 0 & 2 & 10.0 \\
\hline Sem RM & 7 & 33.3 & 10 & 50.0 \\
\hline Total & 21 & 100.0 & 20 & 100.0 \\
\hline
\end{tabular}

Tabela 4

Alterações visuais no G1 e G2 em frequências $e$ percentagens

\begin{tabular}{|c|c|c|c|c|}
\hline \multirow[b]{2}{*}{ Alterações Visuais } & \multicolumn{2}{|c|}{ G1 } & \multicolumn{2}{|r|}{ G2 } \\
\hline & $\begin{array}{l}\text { Freq } \\
\text { (n) }\end{array}$ & $\begin{array}{l}\text { Perc. } \\
(\%)\end{array}$ & $\begin{array}{c}\text { Freq } \\
\text { (n) }\end{array}$ & $\begin{array}{l}\text { Per } \\
(\%)\end{array}$ \\
\hline Estrabismo & 6 & 28.6 & 7 & 35.0 \\
\hline Estrabismo + Hipermetropia & 2 & 9.5 & 0 & 0 \\
\hline Estrabismo + Miopia & 2 & 9.5 & 0 & 0 \\
\hline Estrabismo + Nistagmo & 1 & 4.8 & 1 & 5.0 \\
\hline Miopia & 1 & 4.8 & 0 & 0 \\
\hline Sem Alterações & 9 & 42.9 & 12 & 60.0 \\
\hline Total & 21 & 100.0 & 20 & 100.0 \\
\hline
\end{tabular}

Os três grupos na análise descritiva da tabela 5 caraterizam-se em relação ao desenvolvimento visuopercetivo e da linguagem de forma diferente sendo o G1 mais frágil na área visuopercetiva em relação ao G2 mas não na área da linguagem. Ambos os grupos apresentam percentis inferiores ao G3 de crianças tipicamente desenvolvidas.

Tabela 5

Caraterização da amostra em relação ao desenvolvimento visuopercetivo e linguagem com resultados dos testes em percentis

\begin{tabular}{|c|c|c|c|c|c|c|}
\hline & $\begin{array}{l}\text { Desenvolv. } \\
\text { Visuo } \\
\text { percetivo e } \\
\text { Linguagem }\end{array}$ & $\mathrm{N}$ & Mín & Máx & Média & $\begin{array}{l}\text { Desvio } \\
\text { padrão }\end{array}$ \\
\hline \multirow{4}{*}{ G1 } & WISC III - IOP & 21 & 1 & 65 & 19.05 & 19.449 \\
\hline & F.C.Rey & 21 & 1 & 30 & 12.52 & 8.394 \\
\hline & WISC III - ICV & 21 & 3 & 97 & 42.00 & 27.593 \\
\hline & GOL-E & 21 & 5 & 75 & 27.62 & 22.339 \\
\hline \multirow{4}{*}{ G2 } & WISC III - IOP & 20 & 2 & 82 & 25.45 & 24.334 \\
\hline & F.C.Rey & 20 & 1 & 99 & 27.25 & 24.895 \\
\hline & WISC III - ICV & 20 & 2 & 93 & 51.05 & 31.889 \\
\hline & GOL-E & 20 & 5 & 75 & 23.75 & 22.117 \\
\hline \multirow{4}{*}{ G3 } & WISC III - IOP & 28 & 5 & 99 & 68.31 & 25.384 \\
\hline & F.C.Rey & 28 & 20 & 99 & 80.42 & 25.757 \\
\hline & WISC III - ICV & 28 & 47 & 99 & 83.81 & 15.144 \\
\hline & GOL-E & 28 & 25 & 90 & 67.85 & 17.020 \\
\hline
\end{tabular}

\section{Instrumentos e Procedimentos}

$\mathrm{Na}$ avaliação da leitura com avaliação simultânea do processamento fonológico e visual recorreu-se à tecnologia eye-tracking. Para calibrar e detetar a capacidade de utilização do sistema eye-tracking fez-se um teste ao nível da atenção visual, prévio à avaliação da leitura, permitindo avaliar a fixação e o seguimento visual. Este teste foi adaptado para o computador partindo de um teste utilizado em material físico realizado por Michael Clarke no GAZE Project da UCL - University College London. Nesta avaliação é solicitado ao sujeito a fixação de uma bola vermelha que aparece e desaparece no écran em 4 quadrantes no centro em cima, no centro em baixo, à esquerda e à direita, constituindo um bom ponto de partida na avaliação para se poder usar esta ferramenta.

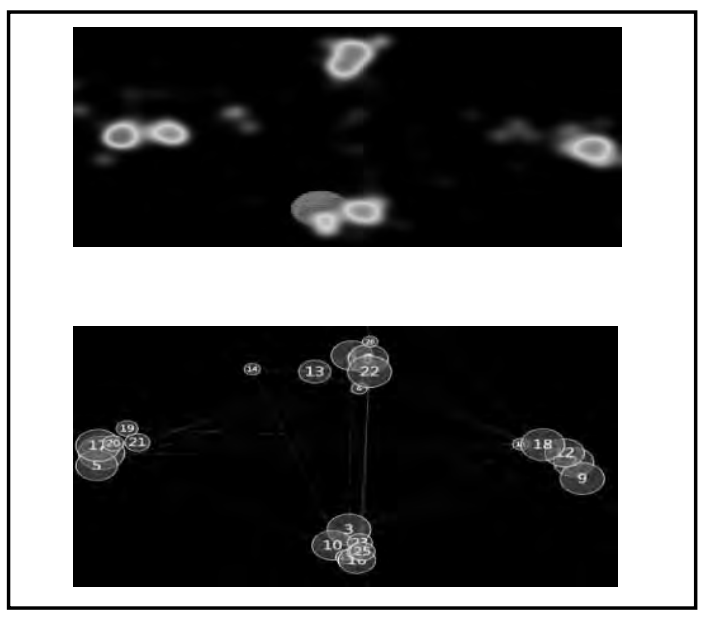

Figura 1 Padrão de fixação e seguimento visual nos quatro quadrantes com sequenciação e mancha de registo de frequência

Procedeu-se à avaliação da leitura tendo sido os três grupos avaliados a nível da Leitura com o teste Avaliação da Leitura em Português Europeu - ALEPE (Sucena \& Castro, 2011), permitindo ler 48 palavras e 24 pseudopalavras no écran do computador. Cada palavra é apresentada durante $10 \mathrm{~s}$ desaparecendo de seguida e surgindo uma nova palavra. O software da ALEPE permite registar a gravação da voz e o tempo de reação sendo este o tempo real de leitura do estímulo em voz alta. Este software regista ainda a cotação da resposta que o examinador realiza clicando o botão do rato do lado esquerdo para a resposta incorreta e lado direito para a resposta correta durante a avaliação. Em simultâneo registou-se a fixação e a sequência do seguimento visual durante a leitura de palavras com outro software Gaze Viewer. Analisou-se o número de fixações e o desenho do seguimento entre fixações durante a leitura da palavra desde a exposição ao estímulo visual até terminar a leitura da palavra.

\section{Resultados}

A qualidade da leitura foi classificada segundo a audição da voz gravada com visualização do espectrograma, gravado no software da ALEPE, em 4 
parâmetros, silábica (silaba a silaba), hesitante (pausa a meio da palavra), corrente (sem pausas) e expressiva (sem pausas e com entoação) com acordo entre observadores (fig.2).

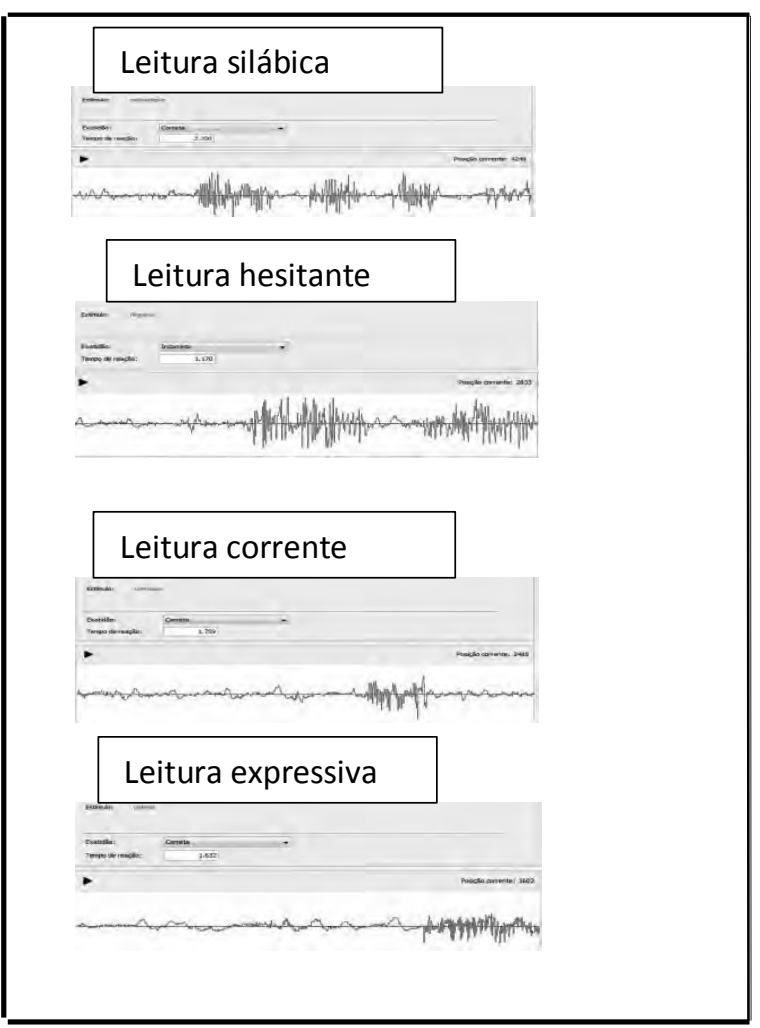

Figura 2 Padrões de leitura do espectrograma da voz gravada

A distribuição da qualidade da leitura nos três grupos encontra-se na tabela 6 evidenciando o G1 maior imaturidade leitura predominantemente silábica.

Tabela 6 .

Qualidade da leitura nos três grupos, distribuição descritiva em frequência e percentagem

\begin{tabular}{lrrrrrr}
\hline \multirow{2}{*}{$\begin{array}{l}\text { Qualidade } \\
\text { da Leitura }\end{array}$} & $\begin{array}{r}\text { Freq } \\
(\mathrm{N})\end{array}$ & $\begin{array}{r}\text { Perc } \\
(\%)\end{array}$ & $\begin{array}{r}\text { Freq } \\
(\mathrm{N})\end{array}$ & $\begin{array}{r}\text { Perc } \\
(\%)\end{array}$ & $\begin{array}{r}\text { Freq } \\
(\mathrm{N})\end{array}$ & \multicolumn{2}{c}{$\begin{array}{l}\text { Perc } \\
(\%)\end{array}$} \\
\hline Silábica & 11 & 52.4 & 7 & 35.0 & 0 & 0.0 \\
Hesitante & 3 & 14.3 & 4 & 20.0 & 0 & 0.0 \\
Corrente & 5 & 23.8 & 9 & 45.0 & 9 & 32.1 \\
Expressiva & 2 & 9.5 & 0 & 0.0 & 19 & 67.9 \\
Total & 21 & 100.0 & 20 & 100.0 & 28 & 100.0 \\
\hline
\end{tabular}

Foram classificados qualitativamente, através da observação dos registos nos vídeos com o software gaze viewer, os padrões de fixação e seguimento visual em cinco parâmetros: ponto único (uma fixação por palavra); linear horizontal (fixações múltiplas ao longo da palavra horizontalmente da esquerda para a direita); ziguezague horizontal (com fixações e regressões horizontais da esquerda para a direita e da direita para a esquerda); ziguezague vertical (com fixações e regressões verticais de cima para baixo e de baixo para cima); ziguezague vertical + horizontal (com fixações e regressões em ambas as direções) com acordo entre observadores ver (fig. 3).

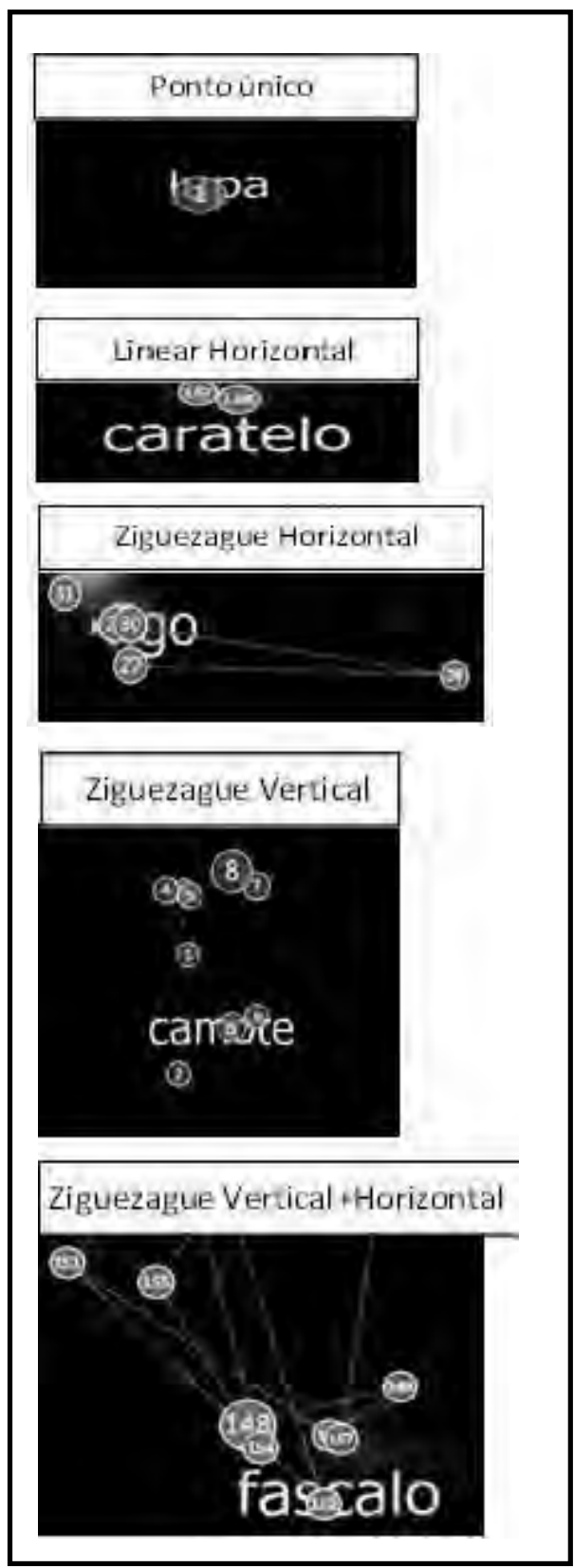

Figura 3 Padrões de leitura de fixação e seguimento visual

Conforme se observa na tabela 7 o G1 mantem um padrão qualitativo de maior dificuldade na coordenação visuomotora com uma grande percentagem do padrão de ziguezague horizontal representando a necessidade de retrocessos visuais na leitura necessitando de um maior $\mathrm{n}^{\mathrm{o}}$ de fixações visuais. O grupo de controlo é o que apresenta uma percentagem com um padrão único e linear horizontal próximo do adulto também com um menor $n^{\circ}$ de fixações. 
Tabela 7.

Padrão de seguimento visual nos três grupos, distribuição descritiva

\begin{tabular}{|c|c|c|c|c|c|c|}
\hline \multirow{2}{*}{$\begin{array}{l}\text { Padrão de } \\
\text { seguimento } \\
\text { visual }\end{array}$} & \multicolumn{2}{|c|}{ G1 } & \multicolumn{2}{|c|}{ G2 } & \multicolumn{2}{|c|}{ G3 } \\
\hline & $\begin{array}{r}\text { Freq } \\
(\mathrm{N})\end{array}$ & $\begin{array}{c}\text { Perc } \\
(\%)\end{array}$ & $\begin{array}{l}\text { Freq } \\
(\mathrm{N})\end{array}$ & $\begin{array}{c}\text { Perc } \\
(\%)\end{array}$ & $\begin{array}{l}\text { Freq } \\
(\mathrm{N})\end{array}$ & $\begin{array}{c}\text { Perc } \\
(\%)\end{array}$ \\
\hline Ponto único & 0 & 0.0 & 0 & 0.0 & 2 & 7.1 \\
\hline $\begin{array}{l}\text { Linear } \\
\text { horizontal }\end{array}$ & 5 & 23.8 & 10 & 50.0 & 18 & 64.3 \\
\hline $\begin{array}{c}\text { Ziguezague } \\
\text { horizontal }\end{array}$ & 15 & 71.4 & 10 & 50.0 & 1 & 3.6 \\
\hline $\begin{array}{l}\text { Ziguezague } \\
\text { vertical }\end{array}$ & 1 & 4.8 & 0 & 0.0 & 5 & 17.9 \\
\hline $\begin{array}{l}\text { Ziguezague } \\
\text { vertical + } \\
\text { horizontal }\end{array}$ & 0 & 0.0 & 0 & 0.0 & 2 & 7.1 \\
\hline Total & 21 & 100.0 & 20 & 100.0 & 28 & 100.0 \\
\hline
\end{tabular}

Foram ainda comparadas as variáveis visuais e de leitura entre três grupos, conforme tabela 8 .

Tabla 8.

Comparações múltiplas das variáveis não paramétricas entre grupos através do teste Kruskal-Wallis

\begin{tabular}{|c|c|c|c|}
\hline Variáveis & Comparação & $p$ & sig \\
\hline \multirow{3}{*}{$\begin{array}{l}\text { No fixação } \\
\text { visuais }\end{array}$} & G1-G2 & 0.297 & ns \\
\hline & G1-G3 & 0.000 & *** \\
\hline & G2-G3 & 0.000 & 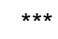 \\
\hline \multirow{3}{*}{$\begin{array}{l}\text { Acertos na } \\
\text { Leitura palavras }\end{array}$} & G1-G2 & 0.957 & ns \\
\hline & G1-G3 & 0.009 & $\star * *$ \\
\hline & G2-G3 & 0.001 & $\star \star \star ~$ \\
\hline \multirow{3}{*}{$\begin{array}{l}\text { Acertos na } \\
\text { Leitura de } \\
\text { pseudopalavras }\end{array}$} & G1-G2 & 0.249 & ns \\
\hline & G1-G3 & 0.004 & 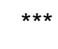 \\
\hline & G2-G3 & 0.145 & ns \\
\hline \multirow{3}{*}{$\begin{array}{l}\text { Tempo de } \\
\text { reação leitura de } \\
\text { palavras }\end{array}$} & G1-G2 & 0.094 & ns \\
\hline & G1-G3 & 0.000 & 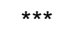 \\
\hline & G2-G3 & 0.071 & ns \\
\hline \multirow{3}{*}{$\begin{array}{l}\text { Tempo de } \\
\text { reação leitura de } \\
\text { pseudopalavras }\end{array}$} & G1-G2 & 0.382 & ns \\
\hline & G1-G3 & 0.001 & $\star \star \star ~$ \\
\hline & G2-G3 & 0.013 & $\star \star$ \\
\hline
\end{tabular}

$* * * p<0,01 ; * * p<0,05$

\section{Discussão}

Os resultados encontrados sugerem a existência de um padrão do $\mathrm{G} 1$, crianças nascidas pré-termo, com maior fragilidade inicial quer na patologia diagnóstica, nas lesões cerebrais e visuais apresentando repercussões mais evidentes no neurodesenvolvimento visuopercetivo. Estes resultados vão no sentido da literatura encontrada em estudos anteriores de seguimento de prematuros (Anderson, 2003; Bhutta, 2002; Guarini, 2010; Litt, 2005; Kellr-Margulis, 2011; Olivieri 2012. Contudo não se encontram diferenças significativas entre G1 e G2 quer ao nível do ${ }^{\circ}$ médio de fixações quer ao nível dos acertos na leitura e tempos de reação, embora com valores mais baixos de percentis no G1 estes não diferem de forma significativa. No entanto este grupos comparados com o grupo de controlo diferem sempre exceto o G2 que tem melhores resultados na leitura de pseudopalavras e nos tempos de reação na leitura de palavras aproximando-se dos resultados obtidos pelo grupo de controlo e não diferindo deste de forma significativa. Podemos admitir que o G1 que apresentou um perfil de maior fragilidade do neurodesenvolvimento percetivo revelou maiores dificuldades na leitura ao nível do processamento fonológico, com uma leitura maioritariamente silábica e nos resultados de acertos de leitura sobretudo das pseudopalavras apresentando ao mesmo tempo também maiores dificuldades no processamento visual registando um padrão de seguimento visual maioritariamente em ziguezague horizontal associado a regressões e um maior $n^{\circ}$ de fixações em relação ao grupo de termo com uma maior diferença em relação ao grupo de controlo em todas as variáveis desta comparação. Parece ser o processamento visuopercetivo um bom indicador das dificuldades na leitura devendo por isso ser também trabalhado em intervenção com treinos específicos nestas populações em idades escolares. Por outro lado o recurso ao material digital, e a este em particular, facilita a realização de avaliações, dando um feedback imediato para onde a criança esteve a olhar e poderá ainda ser facilitador a nível da motricidade complementando o que o papel e lápis não permite a maioria das vezes nesta população com perturbação neuromotora e necessidades educativas especiais.

\section{Bibliografia}

Anderson, P. (2003). Neurobehavioral Outcomes of School-age Children Born Extremely Low Birth Weight or Very Preterm in the 1990s. JAMA, 289(24), 3264. doi:10.1001/jama.289.24.3264

Andrews, J., Ben-Shachar, M., Yeatman, J., Flom, L., Luna, B., \& Feldman, H. (2009). Reading performance correlates with white-matter properties in preterm and term children. Developmental Medicine \& Child Neurology, 52(6), e94-e100. doi:10.1111/j.1469-8749.2009.03456.x

Anjari, M., Srinivasan, L., Allsop, J., Hajnal, J., Rutherford, M., Edwards, A., \& Counsell, S. (2007). Diffusion tensor imaging with tract-based spatial statistics reveals local white matter abnormalities in preterm infants. Neuroimage, 35(3), 1021-1027. doi:10.1016/j.neuroimage.2007.01.035

Bassi, L., Ricci, D., Volzone, A., Allsop, J., Srinivasan, L., \& Pai, A. et al. (2008). Probabilistic diffusion tractography of the optic radiations and visual function in preterm infants at term equivalent age. Brain, 131(2), 573-582. doi:10.1093/brain/awm327

Bax, M., Goldstein, M., Rosenbaum, P., Leviton, A., Paneth, N., \& Dan, B. et al. (2005). Proposed definition and classification of cerebral palsy, April 2005. Developmental Medicine \& Child Neurology, 47(8), 571-576. doi:10.1017/s001216220500112x

Bhutta, A., Cleves, M., Casey, P., Cradock, M., \& Anand, K. (2002). Cognitive and Behavioral Outcomes of School-Aged Children Who Were Born 
$\begin{array}{lll}\text { Preterm. } & \text { JAMA, } & \text { 288(6), }\end{array}$ doi:10.1001/jama.288.6.728

Blythe, H., Liversedge, S., Joseph, H., White, S., \& Rayner, K. (2009). Visual information capture during fixations in reading for children and adults. Vision Research, 49(12), 1583-1591. doi:10.1016/j.visres.2009.03.015

Butcher, P., Bouma, A., Stremmelaar, E., Bos, A., Smithson, M., \& Van Braeckel, K. (2012). Visuospatial perception in children born preterm with no major neurological disorders. Neuropsychology, 26(6), 723-734. doi:10.1037/a0029298

Dambacher, M., Slattery, T., Yang, J., Kliegl, R., \& Rayner, K. (2013). Evidence for direct control of eye movements during reading. Journal Of Experimental Psychology: Human Perception And Performance, 39(5), 1468-1484. doi:10.1037/a0031647

De Bruïne, F., van Wezel-Meijler, G., Leijser, L., van den Berg-Huysmans, A., van Steenis, A., van Buchem, M., \& van der Grond, J. (2010). Tractography of developing white matter of the internal capsule and corpus callosum in very preterm infants. Eur Radiol, 21(3), 538-547. doi:10.1007/s00330-010-1945-x

Dehaene, S., \& Cohen, L. (2011). The unique role of the visual word form area in reading. Trends In Cognitive Sciences, $15(6)$, doi:10.1016/j.tics.2011.04.003

Dehaene, S., Pegado, F., Braga, L., Ventura, P., Filho, G., \& Jobert, A. et al. (2010). How Learning to Read Changes the Cortical Networks for Vision and Language. Science, 330(6009), 1359-1364. doi:10.1126/science. 1194140

Ellis, Andrew (1995) Leitura, Escrita e Dislexia. Artes Médicas: Porto Alegre

Fazzi, E., Signorini, S., La Piana, R., Bertone, C., Misefari, W., \& Galli, J. et al. (2012). Neuroophthalmological disorders in cerebral palsy: ophthalmological, oculomotor, and visual aspects. Developmental Medicine \& Child Neurology, 54(8), 730-736. doi:10.1111/j.1469-8749.2012.04324.x

Guarini, A., Sansavini, A., Fabbri, C., Savini, S., Alessandroni, R., Faldella, G., \& Karmiloff-Smith, A. (2010). Long-term effects of preterm birth on language and literacy at eight years. Journal of child language, 37(04), 865-885.

Hand, C., O’Donnell, P., \& Sereno, S. (2012). WordInitial Letters Influence Fixation Durations during Fluent Reading. Front. Psychology, 3. doi:10.3389/fpsyg.2012.00085

Himmelmann, K., Hagberg, G., \& Uvebrant, P. (2010). The changing panorama of cerebral palsy in Sweden. X. Prevalence and origin in the birth-year period 1999-2002. Acta Paediatrica, 99(9), 13371343. doi:10.1111/j.1651-2227.2010.01819.x

Kay, E. \& Santos, M. E. (2014). Grelha de observação da Linguagem - Nível Escolar - GOL -E. Editora:Oficina Didática, Lisboa.

Keller-Margulis, M., Dempsey, A., \& Llorens, A. (2011). Academic Outcomes for Children Born Preterm: A Summary and Call for Research. Early
Childhood Education Journal, 39(2), 95-102. doi:10.1007/s10643-011-0446-0

Kerr-Wilson, C. O., Mackay, D. F., Smith, G. C. S., \& Pell, J. P. (2012). Meta-analysis of the association between preterm delivery and intelligence. Journal of Public Health, 34(2), 209-216.

Litt, J., Taylor, H., Klein, N., \& Hack, M. (2005). Learning Disabilities in Children with Very Low Birthweight: Prevalence, Neuropsychological Correlates, and Educational Interventions. Journal of Learning Disabilities, 38(2), 130-141. doi:10.1177/00222194050380020301

Olivieri, I., Bova, S., Urgesi, C., Ariaudo, G., Perotto, E., \& Fazzi, E. et al. (2012). Outcome of extremely low birth weight infants: What's new in the third millennium? Neuropsychological profiles at four years. Early Human Development, 88(4), 241-250. doi:10.1016/j.earlhumdev.2011.08.012

O’Reilly, M., Vollmer, B., Vargha-Khadem, F., Neville, B., Connelly, A., \& Wyatt, J. et al. (2010). Ophthalmological, cognitive, electrophysiological and MRI assessment of visual processing in preterm children without major neuromotor impairment. Developmental Science, 13(5), 692-705. doi:10.1111/j.1467-7687.2009.00925.x

Ortibus, E., De Cock, P., \& Lagae, L. (2011). Visual Perception in Preterm Children: What Are We Currently Measuring?. Pediatric Neurology, 45(1), 110. doi:10.1016/j.pediatrneurol.2011.02.008

Palisano, R., Rosenbaum, P., Walter, S., Russell, D., Wood, E., \& Galuppi, B. (1997). Development and reliability of a system to classify gross motor function in children with cerebral palsy. Developmental Medicine \& Child Neurology, 39(4), 214-223. doi:10.1111/j.1469-8749.1997.tb07414.x

Ricci, D., Cesarini, L., Gallini, F., Serrao, F., Leone, D., \& Baranello, G. et al. (2010). Cortical Visual Function in Preterm Infants in the First Year. The Journal Of Pediatrics, 156(4),

doi:10.1016/j.jpeds.2009.10.042

Rosenbaum, P., Palisano, R., Bartlett, D., Galuppi, B., \& Russell, D. (2008). Development of the Gross Motor Function Classification System for cerebral palsy. Developmental Medicine \& Child Neurology, 50(4), 249-253. doi:10.1111/j.14698749.2008.02045.x

Saigal, S., \& Doyle, L. (2008). An overview of mortality and sequelae of preterm birth from infancy to adulthood. The Lancet, 371(9608), 261-269. doi:10.1016/s0140-6736(08)60136-1

Narberhaus, A., Lawrence, E., Allin, M., Walshe, M., McGuire, P., \& Rifkin, L. et al. (2009). Neural substrates of visual paired associates in young adults with a history of very preterm birth: Alterations in fronto-parieto-occipital networks and caudate nucleus. Neuroimage, 47(4), 1884-1893. doi:10.1016/j.neuroimage.2009.04.036

Nazir, T. A., \& Huckauf, A. (2008). The visual skill reading. Single word reading: Cognitive, behavioral and biological perspectives, 25-42.

Rayner, K. (2009). Eye movements and attention in reading, scene perception, and visual search. The 
quarterly journal of experimental psychology, 62(8), 1457-1506.

Rayner, K., Slattery, T., Drieghe, D., \& Liversedge, S. (2011). Eye movements and word skipping during reading: Effects of word length and predictability. Journal Of Experimental Psychology: Human Perception And Performance, 37(2), 514-528. doi:10.1037/a0020990

Rey, A. (2002). Teste de Cópia de Figuras Complexas. Centre de Psychologie Appliquée (Paris). Adaptação

Portuguesa: Departamento de Investigação $e$ Publicações Psicológicas-CEGOC-TEA.

Spinelli, E. \& Ferrand, L.(2005). Psicologia da Linguagem. $O$ escrito e o falado, do sinal à significação. Ed. Instituto Piaget. Lisboa.

Sucena, A., \& Castro, S. L. (2011). ALEPE-Avaliação da Leitura em Português Europeu. Lisboa: CEGOC.

Vigilância Nacional da Paralisia Cerebral aos 5 anos de idade. Crianças nascidas entre 2001 e 2003. Publicação do Programa de Vigilância Nacional da paralisia cerebral: Lisboa

Volpe, J. J. (2009). Brain injury in premature infants: a complex amalgam of destructive and developmental disturbances. The Lancet Neurology, 8(1), 110-124.

Wechsler, D. (2003). Escala de Inteligência para Crianças-Terceira Edição (WISC-III): Manual. Lisboa: Cegoc-Tea.

\section{Agradecimentos}

O primeiro agradecimento é dirigido às crianças por se mostrarem extremamente compreensivas e tolerantes e às famílias que autorizaram a participação dos seus filhos neste estudo e assinaram os respetivos consentimentos informados. A realização deste trabalho envolve a contribuição multiprofissional e participação de diversas pessoas especializadas membros da equipa do Centro de Reabilitação de Paralisia Cerebral Calouste Gulbenkian que de uma forma solicita colaboraram na sua área de especialidade fornecendo o seu parecer e discutindo os resultados obtidos em equipa. Este agradecimento estende-se igualmente ao precioso contributo da Eng. Miriam Azevedo e do Eng. Luís Azevedo pela sua orientação na escolha dos instrumentos na área das tecnologias de informação implicadas neste estudo. 